\title{
Solar wind ion trends and signatures: STEREO PLASTIC observations approaching solar minimum
}

\author{
A. B. Galvin ${ }^{1}$, M. A. Popecki ${ }^{1}$, K. D. C. Simunac ${ }^{1}$, L. M. Kistler ${ }^{1}$, L. Ellis $^{1}$, J. Barry ${ }^{1}$, L. Berger ${ }^{2}$, L. M. Blush ${ }^{3}$, \\ P. Bochsler ${ }^{1,3}$, C. J. Farrugia ${ }^{1}$, L. K. Jian ${ }^{4}$, E. K. J. Kilpua ${ }^{5}$, B. Klecker ${ }^{6}$, M. Lee ${ }^{1}$, Y. C.-M. Liu ${ }^{1}$, J. L. Luhmann ${ }^{7}$, \\ E. Moebius ${ }^{1}$, A. Opitz ${ }^{8}$, C. T. Russell ${ }^{4}$, B. Thompson ${ }^{9}$, R. F. Wimmer-Schweingruber ${ }^{6}$, and P. Wurz ${ }^{3}$ \\ ${ }^{1}$ University of New Hampshire, Durham, NH 03824, USA \\ ${ }^{2}$ Institute for Experimental and Applied Physics, Christian-Albrechts-University Kiel, Kiel, Germany \\ ${ }^{3}$ University of Bern, Bern, Switzerland \\ ${ }^{4}$ Institute of Geophysics and Planetary Physics, UCLA, Los Angeles, CA 90024, USA \\ ${ }^{5}$ Department of Physics, Theoretical Physics Division, University of Helsinki, Finland \\ ${ }^{6}$ Max-Planck-Institut für extraterrestrische Physik, 85741 Garching, Germany \\ ${ }^{7}$ Space Science Laboratory, University of California, Berkeley, CA 94720, USA \\ ${ }^{8}$ Centre d'Etude Spatiale des Rayonnements (CNRS-UPS), 31028 Toulouse, France \\ ${ }^{9}$ National Aeronautics and Space Administration, Goddard Space Flight Center, Greenbelt, MD 20771, USA
}

Received: 15 June 2009 - Revised: 8 September 2009 - Accepted: 9 September 2009 - Published: 13 October 2009

\begin{abstract}
STEREO has now completed the first two years of its mission, moving from close proximity to Earth in 2006/2007 to more than 50 degrees longitudinal separation from Earth in 2009. During this time, several large-scale structures have been observed in situ. Given the prevailing solar minimum conditions, these structures have been predominantly coronal hole-associated solar wind, slow solar wind, their interfaces, and the occasional transient event. In this paper, we extend earlier solar wind composition studies into the current solar minimum using high-resolution (1-h) sampling times for the charge state analysis. We examine 2-year trends for iron charge states and solar wind proton speeds, and present a case study of Carrington Rotation 2064 (December 2007) which includes minor ion ( $\mathrm{He}, \mathrm{Fe}, \mathrm{O}) \mathrm{ki}-$ netic and $\mathrm{Fe}$ composition parameters in comparison with proton and magnetic field signatures at large-scale structures observed during this interval.
\end{abstract}

Keywords. Interplanetary physics (Solar wind plasma; Sources of the solar wind)

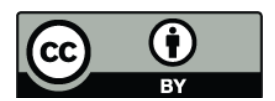

Correspondence to: A. B. Galvin (toni.galvin@unh.edu)

\section{Introduction}

The solar wind is known to have different types of flows originating from different solar sources. The reader is referred to Wimmer-Schweingruber (2002) and Schwenn (2006), and references therein, for a full overview of our recent state of knowledge on solar wind and its sources. In his review, Schwenn (2006) presents four categories of solar flows: (1) fast solar wind in high-speed streams originating from coronal-hole regions, (2) slow solar wind emerging from the non-active Sun near the heliospheric current sheet (HCS) above helmet streamers and their underlying active regions, (3) slow solar wind during high solar activity, merging above active regions, and (4) solar wind explosively expelled from the Sun during coronal mass ejections (CME). The solar sources and relative dominance of these types of flows depend on the phase of the solar cycle. In solar minimum conditions, the majority of solar wind would be expected to fit into categories (1) and (2), but the other flow types would not be excluded.

Solar wind elemental and charge state composition has become a valued tool in distinguishing types of solar wind (coronal hole-associated high-speed streams, ICMEs, and the slow solar winds) and in identifying their solar sources. Typically long-term averages (e.g., von Steiger et al., 2000) or epoch studies (e.g., Geiss et al., 1995) have been used, except for a few case studies.

It has been shown in many such studies that ionic charge (Q) distributions of solar wind minor ions track each other

Published by Copernicus Publications on behalf of the European Geosciences Union. 
and can often be used to characterize flow type. This is most noticeable for interplanetary coronal mass ejection (ICME) events, where in-ecliptic oxygen charge states often extend to +8 and iron charge states often extend to +16 (Galvin, 1997, and references therein; Reinard, 2005, 2008). Von Steiger et al. (2000) studied fast and slow solar wind charge states using Ulysses SWICS data, during portions of the in-ecliptic and out-of-ecliptic mission phases (1992-1998). Their survey concentrated on long-term (300-day accumulation) elemental and charge state abundances of several minor ion species in quasi-stationary flows (stream interaction regions were excluded). Their study confirmed and enlarged previous results by Galvin et al. (1992) for silicon and by Ipavich et al. (1992) for iron, specifically that $\mathrm{Si}$ and $\mathrm{Fe}$ charge states track those of oxygen (positive correlation), and that these two elements $(\mathrm{Si}, \mathrm{Fe})$ in particular have charge states whose "bulk" distributions show distinct but modest differences between coronal hole-associated and slow solar wind. The von Steiger et al. (2000) study also confirmed previous results (Galvin et al., 1992; Ipavich et al., 1992) that for the slow solar wind there are contributions "added on" from higher charge states for both $\mathrm{Si}$ and $\mathrm{Fe}$. In fact, while the average charge state for slow solar wind was found to be slightly higher than for fast solar wind, the von Steiger et al. "bulk" results for Fe in polar coronal holes indicated dominant charges peaking around $+9,+10,+11$, while that of their slow solar wind sample actually shifted slightly toward lower charge states, +8 through +11 (Plate 5 of von Steiger et al., 2000).

Several other studies have concentrated on using composition as a tool in identifying boundaries in large-scale structures, particularly at corotating or stream interaction regions (CIR or SIR). Most of these studies utilized elemental abundance ratios and oxygen charge states ratios as composition markers (Wimmer-Schweingruber et al., 1997, 1999; Zurbuchen et al., 1999). Aellig et al. (1998) extensively used iron charge states in their study.

In this paper we present the analysis of solar wind protons and iron ions over the time interval 14 February 2007 through 31 May 2009 as observed by the STEREO A (STA) observatory, using one-hour sampling intervals. The heliospheric distance of the STEREO A spacecraft is slightly within $1 \mathrm{AU}$, for the interval of this study, typically $0.96-0.97 \mathrm{AU}$. The heliographic latitude varied by \pm 7 degrees (due to the tilt of the solar equator to the ecliptic plane). The separation angle from Earth progressively ranged from $0.7^{\circ}$ (February 2007 ) to $50.4^{\circ}$ (May 2009). The time period covered Carrington Rotations 2053.3 through 2083.9, as calculated at the location of the STEREO A spacecraft. For a selected interval (Sect. 4.3), STEREO B data are also presented. General trends in STEREO B data are similar to those of STEREO A.

During this time the Sun was at or near solar minimum conditions, for what may turn out to be an unusually quiet and extended solar minimum period (e.g., McComas et al., 2008; Luhmann et al., 2009; Leitner et al., 2009).

\section{Data analysis}

The data presented in this study are from the Plasma and Suprathermal Ion Composition (PLASTIC) and the In situ Measurements of Particles And CME Transients (IMPACT) investigations of the Solar Terrestrial Relations Observatory A (STEREO A), covering the first two years of the STEREO mission, February 2007 through May 2009. These instruments are described by Galvin et al. (2008) and Luhmann et al. (2008), respectively.

PLASTIC is a mass and mass-per-charge spectrometer that measures positive ions in the energy-per-charge range of 0.3$86 \mathrm{keV} / \mathrm{e}$, with a one-minute cadence for traversing the 128 logarithmically-spaced E/Q steps (Galvin et al., 2008). Solar wind proton parameters provided by PLASTIC include bulk speed, density, kinetic temperature, and flow direction as described by Simunac (2009). Minor ion parameters include bulk speed, kinetic temperature, flow direction, species and charge state identification.

Solar wind species of different mass are distinguished using the time-of-flight and energy measurements of the PLASTIC mass spectrometer. The TOF and total energy measurements are made after the ions have dropped through a potential of $-20 \mathrm{kV}$. This acceleration boosts the energy of the ions sufficiently to exceed the solid-state detector thresholds. Each elemental species follows its own track in Energy-TOF matrix space (see Fig. 23 of Galvin et al., 2008), allowing one to create different rates corresponding to slices in "mass" space. After mass separation, the mass-per-charge is determined from the measured time-of-flight and selected energyper-charge for each individual event within the selected mass range. The individual events are then binned into $\mathrm{M} / \mathrm{Q}$ rates and normalized to the full sample. These $\mathrm{M} / \mathrm{Q}$ rate bins are used to determine the average charge state and bulk speeds. Systematic errors can be introduced through the choice of the species rate bins and efficiency factors. For iron, these are estimated to be on the order of half a charge state unit, potentially affecting absolute values of the average charge state, but not the general trend information provided here. Species identification techniques are further described by Galvin et al. (2008) and Daoudi et al. (2009).

The solar wind section of the instrument has two different geometrical factors. The "small" geometrical factor is intended for high fluence, such as solar wind protons and alpha particles, while the "main" geometrical factor is intended for species with low abundances. The two geometrical factors share the same electrostatic analyzer. At the start of the E/Q stepping sequence (at $86 \mathrm{keV} / \mathrm{e}$ ) the main geometrical factor is active. As the E/Q steps down through the solar wind, a count rate is monitored to check for fluence levels. The geometrical factor is switched from main to small by an electrostatic "gate" that acts to suppress the fluence after the count rate threshold has been exceeded. This count rate threshold can be adjusted by command, and is set such that the bulk of the solar wind protons is normally within 

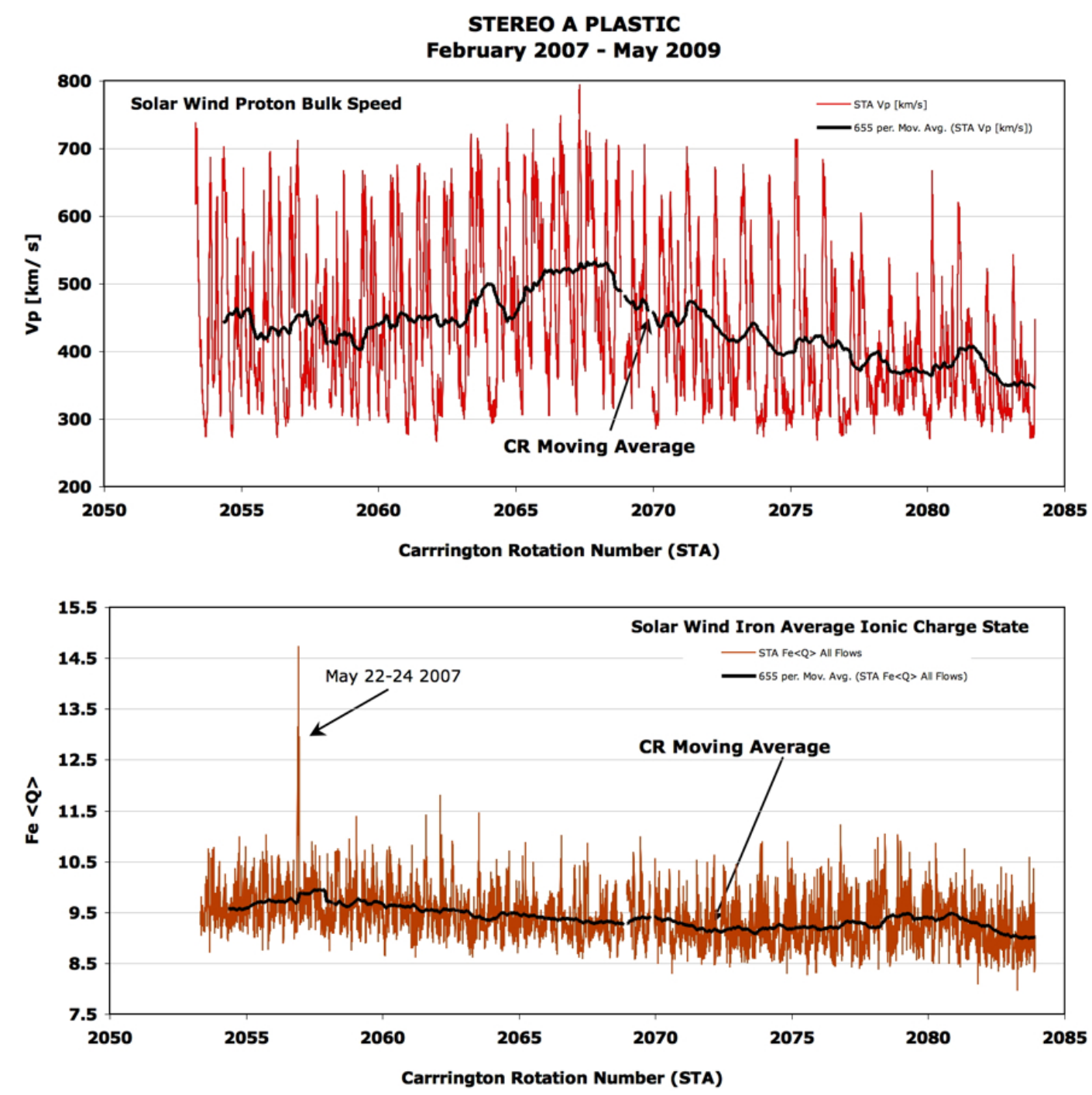

Fig. 1. Solar wind proton speeds (top panel, red trace) and average iron charge states (bottom panel, brown trace) from STEREO A (STA) PLASTIC, covering the time period mid February 2007 through May 2009. Data are one-hour averages. Superimposed on the data is a 655-h moving average (black traces), which approximately corresponds to a nominal synoptic Carrington Rotation interval.

the small geometrical factor. Protons at higher energies, before the threshold is tripped, are measured with the main geometrical factor. In some instances (particularly for helium and oxygen), a solar wind species measurement cadence may have its higher E/Q steps measured with the main geometrical factor, and the lower E/Q steps in the small geometrical factor.

Particular emphasis has been given in this paper to the solar wind iron measurements by PLASTIC. All iron events fall within the "main" channel of the instrument, that is, within a single large geometrical factor, thereby simplifying their analysis and also providing excellent statistics. (Oxygen measurements, which can include contributions from both geometrical factors, will be presented in a future study.) Contributions to the iron mass selection from neighboring species (such as silicon and sulfur) were minimized through an appropriate choice of mass range. Second-order contributions can be further corrected through detailed fitting routines of a full mass-per-charge spectral analysis, but this was not done for the current study. The energy per charge range analyzed for minor ions was $0.7-33 \mathrm{keV} / \mathrm{e}$ to cover solar wind energies. Iron events receive the highest priority in the PLASTIC telemetry and consequently have excellent sampling statistics. For the current study, one-hour accumulations (with 10-min normalizations) were utilized for the minor ion measurements. One-hour event periods with less than 20 counts were excluded (this selection criteria is implemented for statistical reasons, but might engender a bias against rarefaction regions). After the removal of data due to telemetry gaps, instrument operations, and statistical (20 counts) constraints, 


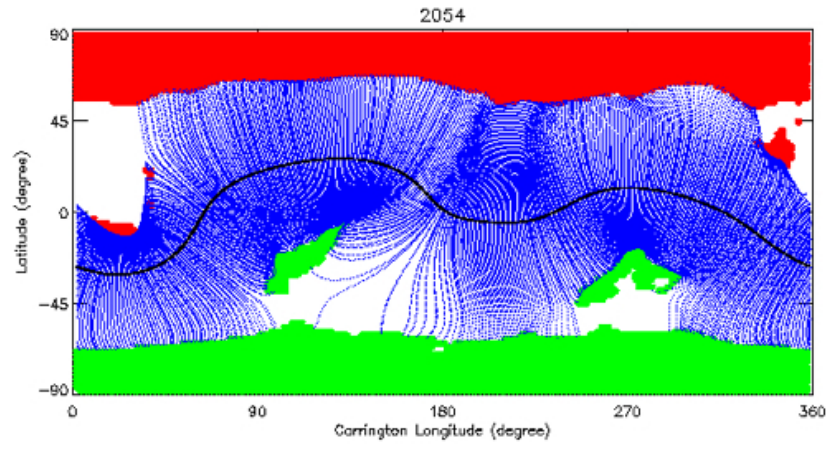

2064

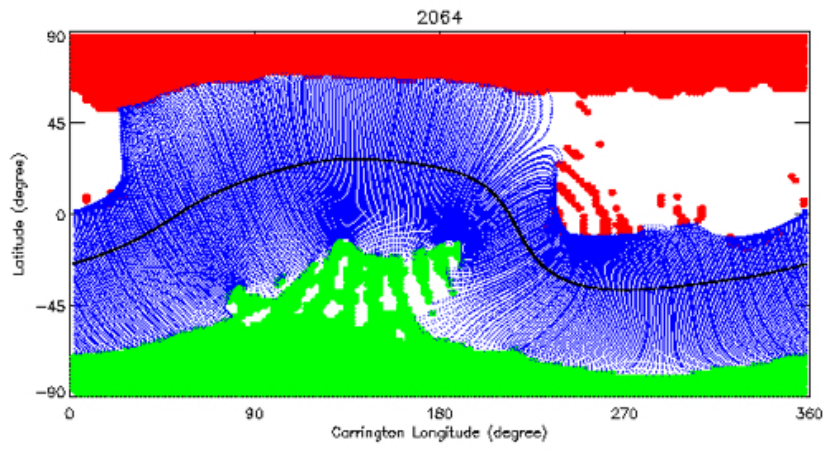

2074
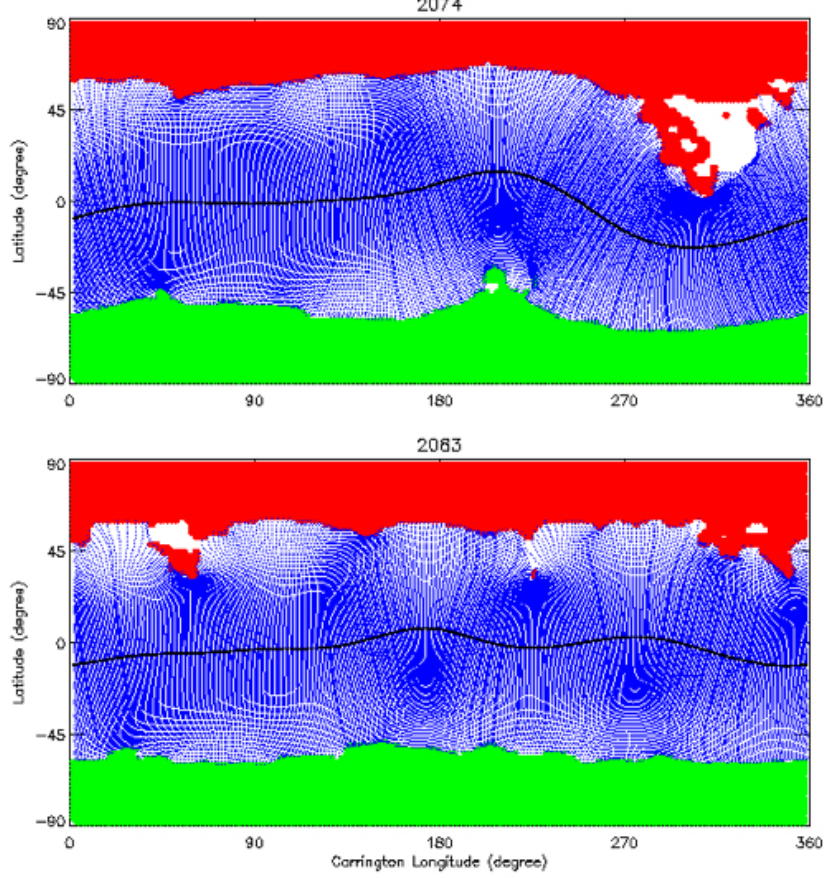

Fig. 2. Selected snapshots of NSO/GONG synoptic coronal hole plots (Latitude versus Carrington Longitude) for Carrington Rotations during the course of the present survey period (dates covered by these rotations can be found in Table 1 for STEREO A). Patches of green and red color indicate the location of the coronal holes and their magnetic polarity (green denotes positive polarity, red negative polarity). The neutral line, which may be identified with the equatorial streamer belt, is drawn in black. the selected analyzed data set constituted over 19000 onehour samples.

Solar wind large-scale structures in this study, such as shocks, stream interfaces, and ICMEs observed by STEREO, have been identified using IMPACT magnetic field and solar wind electron data and PLASTIC solar wind proton data.

\section{Observations: temporal trends in the solar wind and solar corona}

The time series for solar wind proton speeds (1-h averages) covering the survey interval 14 February 2007 through 31 May 2009 is shown in the top panel of Fig. 1. As seen in the figure, the solar wind is dominated by a series of alternating high and low speed solar wind flows, as expected for solar minimum conditions. Overlaid on the time series is a 655 -h moving average, roughly corresponding to one Carrington Rotation (CR). The trend analysis indicates that the average speeds over a given Carrington Rotation, $<V p>_{C R}$, were typical values and relatively steady during the first 10 Carrington Rotations $\left(<V p>_{C R}\right.$ ranged from 423 to $454 \mathrm{~km} / \mathrm{s}$ for Carrington Rotation number 2053 through Carrington Rotation number 2062; see Table 1), peaked during CR $2067\left(<V p>_{C R 2067} \sim 525 \pm 120 \mathrm{~km} / \mathrm{s}\right)$, and thereafter showed a quasi-steady decline to the end of the survey period $\left(<V p>_{C R 2082} \sim 350 \pm 60 \mathrm{~km} / \mathrm{s}\right)$.

The bottom panel of Fig. 1 is the same time series for the derived average charge state for solar wind iron $(\mathrm{Fe}<\mathrm{Q}>$ ). The iron average charge state was also relatively steady for the first ten CRs, but then began to modestly decline. The difference from the start of the reporting period and the end is in excess of half a charge state unit (see Table 1).

One event period in the bottom panel that stands out in the iron $\mathrm{Fe}<\mathrm{Q}>$ data: 22-24 May 2007. This corresponds to two interplanetary coronal mass ejections (ICMEs) that were observed simultaneously by the STEREO A, B, and nearEarth spacecraft.

There was also evolution in the solar sources of solar wind during the survey period. We turn in Fig. 2 to the full integral synoptic coronal-hole maps provided courtesy of NSO Global Oscillation Network Group (GONG). One can see in the figure the evolution of the polar coronal holes, their extensions, isolated equatorial coronal holes, and the neutral line through this two-year period approaching solar minimum. In particular, there is a gradual retreat of the large extensions of the polar coronal holes, and the disappearance of the isolated equatorial holes. The number of active regions/day generally decreased as the solar minimum is approached. One also sees an evolution in the neutral line (and by extension, the equatorial streamer belt) from a sinusoidal pattern within $\pm 40^{\circ}$ band at the start of the survey period, toward a more flat configuration. This in turn leads to a less tilted heliospheric current sheet. See Kilpua et al. (2009b) 
Table 1. Temporal trends in proton speed and iron average charge state (all samples).

\begin{tabular}{|c|c|c|c|c|c|}
\hline CR & Dates (STEREO A) & $<V p>_{C R}$ & Std Dev $<V p>_{C R}$ & $\mathrm{Fe}<\mathrm{Q}>_{C R}$ & Std Dev Fe $<\mathrm{Q}>_{C R}$ \\
\hline $2053 p$ & 14 Feb 07-4 Mar 07 & 445.8 & 134.8 & 9.62 & 0.37 \\
\hline 2054 & 4 Mar 07-31 Mar 07 & 450.8 & 114.0 & 9.60 & 0.36 \\
\hline 2055 & 31 Mar 07-28 Apr 07 & 426.3 & 84.4 & 9.72 & 0.40 \\
\hline 2056 & 28 Apr $07-25$ May 07 & 452.2 & 129.0 & 9.88 & 0.79 \\
\hline 2057 & 25 May 07-21 Jun 07 & 432.2 & 104.4 & 9.72 & 0.36 \\
\hline 2058 & 21 Jun 07-19 Jul 07 & 423.4 & 77.4 & 9.72 & 0.43 \\
\hline 2059 & 19 Jul 07-15 Aug 07 & 438.7 & 105.7 & 9.67 & 0.41 \\
\hline 2060 & 15 Aug 07-12 Sep 07 & 454.0 & 105.4 & 9.60 & 0.38 \\
\hline 2061 & 12 Sep 07-9 Oct 07 & 452.0 & 117.1 & 9.54 & 0.37 \\
\hline 2062 & 9 Oct $07-5$ Nov 07 & 437.5 & 122.5 & 9.52 & 0.41 \\
\hline 2063 & 5 Nov 07-3 Dec 07 & 499.5 & 120.4 & 9.37 & 0.38 \\
\hline 2064 & 3 Dec 07-30 Dec 07 & 457.1 & 123.2 & 9.45 & 0.37 \\
\hline 2065 & 30 Dec 07-27 Jan 08 & 514.7 & 115.2 & 9.39 & 0.31 \\
\hline 2066 & 27 Jan $08-23$ Feb 08 & 520.2 & 115.1 & 9.36 & 0.35 \\
\hline 2067 & 23 Feb 08-21 Mar 08 & 525.9 & 119.4 & 9.34 & 0.41 \\
\hline 2068 & 21 Mar 08-18 Apr 08 & 479.7 & 114.4 & 9.30 & 0.34 \\
\hline 2069 & 18 Apr $08-15$ May 08 & 457.3 & 83.1 & 9.42 & 0.46 \\
\hline 2070 & 15 May $08-12$ Jun 08 & 441.7 & 103.6 & 9.27 & 0.39 \\
\hline 2071 & 12 Jun 08-9 Jul 08 & 451.4 & 106.1 & 9.14 & 0.31 \\
\hline 2072 & 9 Jul 08-5 Aug 08 & 414.2 & 92.1 & 9.22 & 0.40 \\
\hline 2073 & 5 Aug 08-2 Sep 08 & 423.7 & 114.3 & 9.18 & 0.40 \\
\hline 2074 & 2 Sep 08-29 Sep 08 & 398.8 & 105.9 & 9.18 & 0.41 \\
\hline 2075 & 29 Sep $08-27$ Oct 08 & 421.9 & 124.8 & 9.18 & 0.40 \\
\hline 2076 & 27 Oct $08-23$ Nov 08 & 404.2 & 111.7 & 9.32 & 0.48 \\
\hline 2077 & 23 Nov 08-20 Dec 08 & 395.7 & 85.2 & 9.21 & 0.44 \\
\hline 2078 & 20 Dec $08-17$ Jan 09 & 367.2 & 52.9 & 9.45 & 0.52 \\
\hline 2079 & 17 Jan 09-13 Feb 09 & 368.0 & 44.1 & 9.40 & 0.37 \\
\hline 2080 & 13 Feb 09-13 Mar 09 & 388.3 & 76.7 & 9.47 & 0.38 \\
\hline 2081 & 13 Mar 09-9 Apr 09 & 388.3 & 81.0 & 9.24 & 0.40 \\
\hline 2082 & 9 Apr 09-6 May 09 & 350.5 & 58.1 & 9.03 & 0.33 \\
\hline $2083 p$ & 6 May 09-31 May 09 & 350.1 & 60.7 & 9.02 & 0.39 \\
\hline
\end{tabular}

$\mathrm{p}=2053$ and 2083 cover partial CR time intervals.

for details regarding solar structures in 2007-2008 and their impact on STEREO in situ ICME observations.

\section{Observations: $\mathrm{Fe}<\mathrm{Q}>$ in different solar wind flows}

\subsection{Transient and ICME $\mathrm{Fe}<\mathrm{Q}>$ signatures in solar minimum conditions}

In Fig. 3, the data set of solar wind iron average charge states is presented as a scatter plot against the corresponding proton bulk speed. The $\mathrm{Fe}<\mathrm{Q}>$ charge states corresponding to the 22-24 May 2007 ICMEs stand out above any other periods, including the other transient events marked in the figure.

The events labeled as "small transients" have been identified by Kilpua et al. (2009a). These small-scale transients are magnetic structures embedded within the slow solar wind that appear to originate from the vicinity of sector boundaries on the Sun. The authors noted that they might be asso- ciated with the coronal "blobs" first reported by Sheeley et al. (1997).

The remaining ICMEs marked on Fig. 3 are taken from the ICME list compiled by Jian (2009) and from the ICMEs identified by Kilpua et al. (2009b). The Kilpua et al. events are all associated with field strength $>5 \mathrm{nT}$, have an organized magnetic field structure (although not necessarily magnetic cloud), and a duration of at least $3 \mathrm{~h}$. These ICMEs are grouped by the authors according to whether they are compressed in the leading edge of a high-speed stream, or surrounded by slow solar wind flows. These groupings are marked in Fig. 3.

Regarding the "small-scale transients", these have always in the past been treated within the "catch-all" slow solar wind label, and therefore it is not surprising that their charge states fall within the same nominal $\mathrm{Fe}<\mathrm{Q}>$ range for slow solar wind. The most remarkable aspect of the Fe average charge in the remaining ICMEs is that they are not remarkable. With 


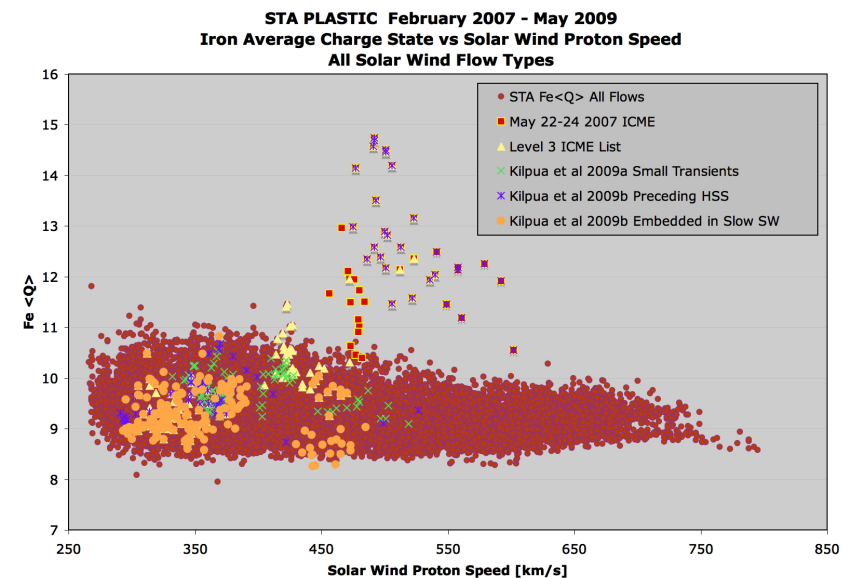

Fig. 3. Solar wind iron average charge states observed by STEREO A (STA) compared against solar wind speeds covering 27.5 months from 14 February 2007 through 31 May 2009. Each data point (brown circles) represents an hour sampling interval. Also shown are time periods identified by various sources (Jian, 2009; Kilpua et al., 2009a, b) as transient or ICME intervals. (There is overlap among some of the identified transient periods, for example as seen in the 22-24 May 2007 intervals.)

the notable exception of the events encompassing the 22-24 May 2007 ICMEs, all of the iron average charge states for the ICMEs and small transients shown here fall within the range $8-11$; that is, the same range seen for the slow solar wind.

As pointed out by Galvin (1997), Galvin and Gloeckler (1997), Reinard (2008) and others: while high ionization states are indicative that the flow is an ICME, the converse is not true: ICMEs run the full gauntlet of solar wind average charge states, including nominal values. In the current survey, none of the identified events meet the Fe charge " $\geq 16$ " criteria set forth in some ICME studies (e.g., Richardson and Cane, 2004). However, some ICME events exhibit a "locally" significant increase in charge state. This can be seen for the 22-24 May 2007 events (Figs. 1, 3), and in other events in the survey. For example, the ICME event on 19 November (22:00 UT) to 21 November (03:17 UT) 2007 has been identified by Jian (2009) based on field rotation and bidirectional electron flux (BDE). As seen Fig. 4, the Fe average charge state is "locally" high when compared with the $\mathrm{Fe}$ $<\mathrm{Q}>$ observed in the ambient solar wind over the remainder of the month, even though this event does not stand out in Fig. 1.

The 22-24 May 2007 ICME events were seen early in the survey interval, when there was more solar activity, and are distinguished by having the highest $\mathrm{Fe}<\mathrm{Q}>$ measured to date by STEREO A. These events were associated with the central passage of solar active region 10956, which was a low latitude (i.e., near ecliptic) active region. This region produced several small flares and more than one CME. The asso-

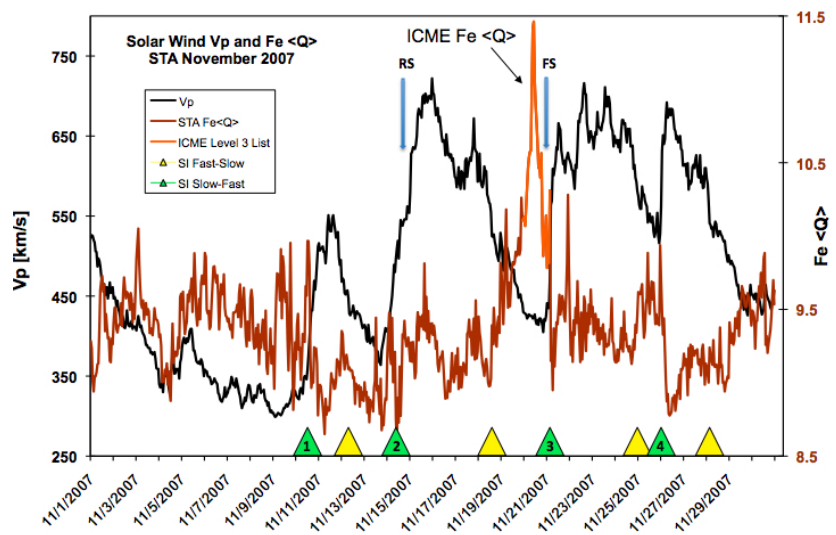

Fig. 4. During November 2007, coronal hole-associated streams, slow solar wind, and an ICME were observed by STEREO A (STA). The black trace is the solar wind proton speed, while the brownorange trace is the iron average charge state. The orange portion of the $\mathrm{Fe}<\mathrm{Q}>$ trace corresponds to the ICME interval identified by Jian (2009). This interval stands out compared to the $\mathrm{Fe}<\mathrm{Q}>$ for other flow types ( $\mathrm{CH}$ or slow) during this particular month, but not when compared to overall averages for the entire survey. The figure shows markers for the solar wind stream interfaces as determined using the entropy criteria described by Simunac (2009). The green arrowheads mark stream interfaces with an increase in entropy (nominally the "slow to fast" or SIR transition, although in case 3 it is also for the slow/ICME to $\mathrm{CH}$ transition), while the yellow arrowheads mark the drop in entropy (nominally the "fast to slow" solar wind transition). Shown near the top as blue arrows are the reverse and forward shocks identified by Jian (2009).

ciated magnetic clouds observed by STEREO A, STEREO B and Wind spacecraft have been extensively discussed in the literature (see Kilpua et al., 2009c). Reconstruction using the Grad-Shafranov method indicates STEREO A had a near central passage through the 23 May magnetic cloud (Möstl et al., 2009).

The more nominal charge states we observe for the other ICMEs reported by Kilpua et al. (2009b) and Jian (2009) may be related to their general weak (modest magnetic field strength) nature and/or their solar origins. Reinard (2005, 2008) produced a comprehensive study of eight years (19982006) of in-ecliptic ICMEs signatures using ACE data, particularly compositional signatures. She found compositional enhancements (or lack thereof) were linked to the energetics of the originating solar event (as in flare magnitude), the location within the ICME based on the solar source longitude, and the magnetic structure of the ICME. For the specific parameter of interest here, she found that a range of $\mathrm{Fe}$ $<\mathrm{Q}>$ between $\sim 10$ to 16 , and determined a positive correlation between higher $\mathrm{Fe}<\mathrm{Q}>$ values and events associated with a large flare, events originating in central longitudes, and events with the magnetic structure identified as a magnetic cloud. 
The $\mathrm{Fe}<\mathrm{Q}>$ values we observe here may also reflect a unique aspect of solar minimum conditions in the ecliptic plane, due to the propagation of high latitude ICMEs. In the latter part of the survey period, CMEs originating from high latitude active regions and polar crown filament eruptions were deflected toward the ecliptic, as reported by Kilpua et al. (2009b). They suggest such events may be a significant contribution to the ICMEs observed by STEREO. If so, another parameter that should to be included in characterizing the observed $\mathrm{Fe}<\mathrm{Q}>$ is the latitude of the solar source. Galvin (1997) and Galvin and Gloeckler (1997) reported a distinctive latitudinal variation in the ICME solar wind minor ion charge states observed in situ by Ulysses SWICS in the time frame $1992-1994$ (corresponding to $8^{\circ}$ to $61^{\circ}$ southern latitudes, in the declining phase of solar cycle 22). These Ulysses ICMEs showed a systematic transition in the charge state distributions of oxygen, silicon, and iron as the space probe's latitude increased. In the case of iron, the dominant charge state changed from +16 in-ecliptic to +10 to +11 at the higher latitudes. Lemen (1996) showed that some of these Ulysses high latitude events came from arcade events associated with coronal-hole changes. If the current solar minimum conditions are causing the deflection of high latitude CMEs down towards the ecliptic, this may account, at least in part, for the relatively modest average charge states observed in these solar minimum ICMEs.

\subsection{Slow and fast solar wind signatures in solar mini- mum conditions}

Figure 4 shows the solar wind $\mathrm{Fe}<\mathrm{Q}>$ in a time interval (November 2007) that contained the full spectrum of solar wind flow types: an ICME, four coronal hole-associated $(\mathrm{CH})$ high speed streams, slow solar wind, and their associated stream interfaces (slow-to-fast, fast-to-slow). The period 13-16 November 2007, with its slow-to-fast stream interface, has been extensively reported upon by Jian et al. (2009) in a multi-spacecraft study involving STEREO A (STA), STEREO B (STB), and the Advanced Composition Explorer (ACE). Concentrating here on the $\mathrm{Fe}<\mathrm{Q}>$ features, one notices a local drop in $\mathrm{Fe}<\mathrm{Q}>$ at the locations marked as "slow to fast" stream interface; that is, when a fast stream overtakes a slow stream, conventionally termed an SIR or CIR (see Jian et al., 2009). These are easiest to see for the third and fourth SIRs (green markers). In general, the transitions in $\mathrm{Fe}<\mathrm{Q}>$ at the fast-to-slow stream interface are less articulated. This result has been reported before (e.g., Zurbuchen et al., 1999). This effect may at least partly be a consequence of propagation effects - the slow-to-fast SIR occurs in the compression region, while the fast-to-slow transition occurs in the rarefaction region, but other processes have been suggested (e.g., Fisk et al., 1998).

What is clear from the figure is that there is quite a bit of structure within the slow and even within the $\mathrm{CH}$ solar wind, and as expected, the $\mathrm{Fe}<\mathrm{Q}>$ in the slow and $\mathrm{CH}$ solar winds are similar, but with the average charge state somewhat lower in the $\mathrm{CH}$ solar wind (Aellig et al., 1998).

In Fig. 5, identified transient (ICME) periods have been removed, and the remaining data samples are displayed as a contour plot in number of samples, again with $\mathrm{Fe}<\mathrm{Q}>$ plotted against $V p$. This new data set constitutes over 19255 one-hour samples of coronal hole-associated ("fast") and slow solar wind, as well as their stream interfaces. This data set is summarized in Table 2.

Coronal holes (polar, extensions, and isolated holes) are the acknowledged source of the high-speed solar wind (e.g., Cranmer, 2002; Isenberg, 2001; Lindblad, 1990). The slow solar wind has more than one origin. More than half of the non-ICME data samples presented in Fig. 5 have wind speeds below $450 \mathrm{~km} / \mathrm{s}$. We recognize that the lower speed solar wind is not solely constituted of "slow" solar wind in the conventional sense of slow wind within the heliospheric plasma sheet and of streamer belt origin. The "slow" speed samples presented here include lower speed $\mathrm{CH}$ associated solar wind, and high-speed $\mathrm{CH}$ solar wind that has been modified ("braked") within stream interaction regions (SIRs). Similarly, pressure gradients at stream interaction regions can accelerate the slow solar wind that is ahead of high-speed streams (Gosling, 1996). Therefore, one expects some mixing in the solar wind characteristics when simplistically categorizing by speed. Nonetheless, although the identification of the source of every stream parcel is not applied here, one can see certain trends (Fig. 5 and Table 2):

- The majority of solar wind samples are low speed, with $62 \%$ below $450 \mathrm{~km} / \mathrm{s}$, and $47 \%$ below $400 \mathrm{~km} / \mathrm{s}$. Only $12 \%$ of the data set were characterized by speeds $>600 \mathrm{~km} / \mathrm{s}$, and less than $1 \%$ by speeds $>700 \mathrm{~km} / \mathrm{s}$ (see insert at bottom of Fig. 5).

- The largest sample size occurs in speed bin 300$350 \mathrm{~km} / \mathrm{s}$. A small hump for the high-speed cases occurs in speed bin $600-650 \mathrm{~km} / \mathrm{s}$.

- The average iron charge state over the entire population is $<\mathrm{Q}>\sim 9.4$, with a standard deviation of the population of 0.4. The range is $<\mathrm{Q}>\sim 8$ to 12 . This range is fully represented at the lower speeds $(<450 \mathrm{~km} / \mathrm{s}$ ). (See insert at bottom of Fig. 5.)

- For 56 stream interfaces at STEREO A, as identified by using the total pressure (Jian, 2009) and covering the period March 2007-September 2008, the average proton speed for the hourly samples containing the identified stream interface period was $454 \pm 79 \mathrm{~km} / \mathrm{s}$, with a median value of $445 \mathrm{~km} / \mathrm{s}$. More than $96 \%$ had speeds below $600 \mathrm{~km} / \mathrm{s}$, and none were above $660 \mathrm{~km} / \mathrm{s}$. These interface samples are expected to constitute a mixture of (accelerated) slow solar wind and (decelerated) highspeed stream. 


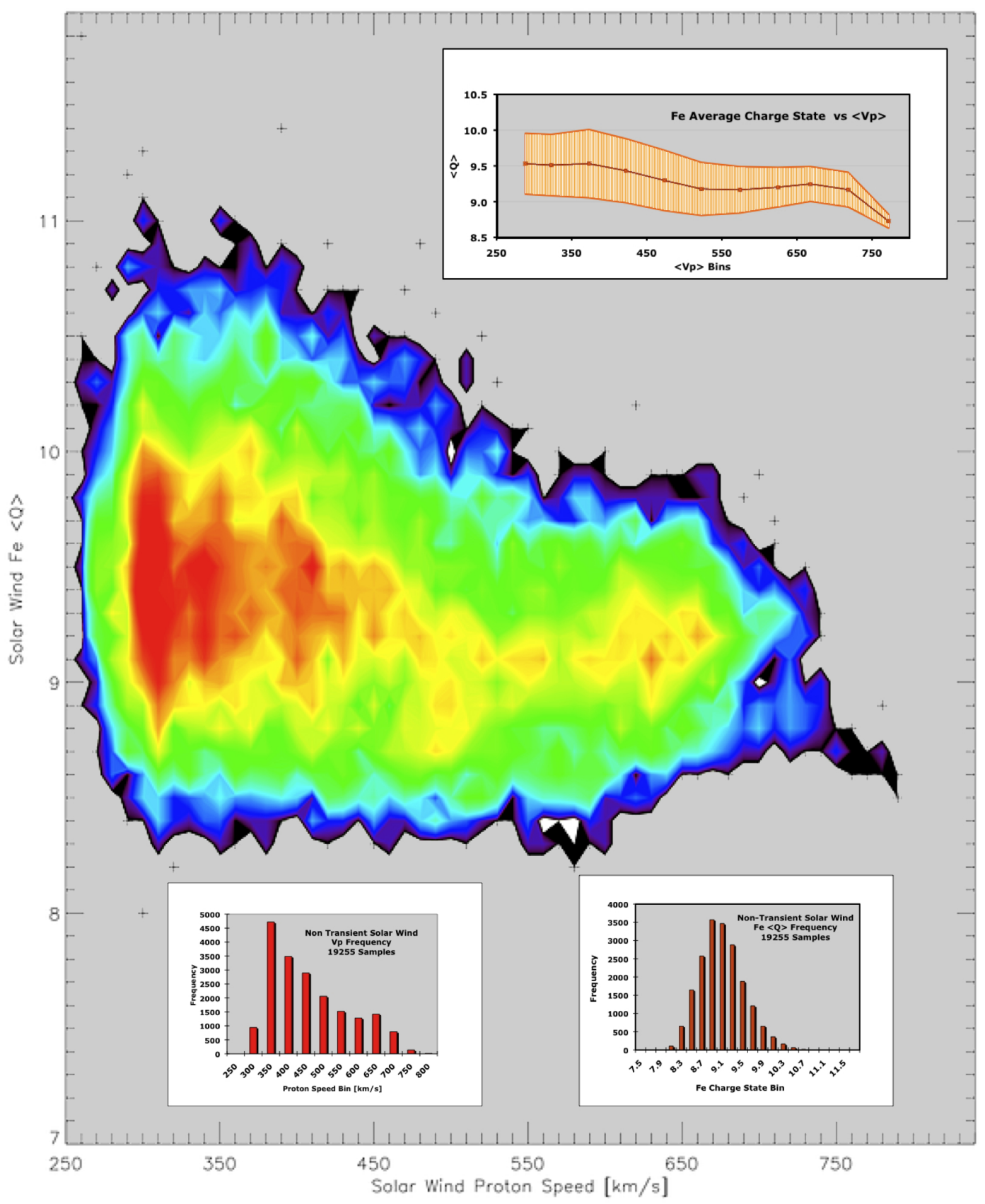

Fig. 5. Same data as Fig. 3, but the identified transient periods have been removed and the data are displayed as a contour plot in number of samples. The non-transient event data set constitutes over 19255 one-hour samples of fast and slow solar wind. Shown as inserts are: (Top) the average iron charge state per velocity bin with \pm 1 -sigma of the population as a function of solar wind speed. (Bottom left) Frequency distribution of solar wind speeds, $50 \mathrm{~km} / \mathrm{s}$ binning. (Bottom right) Frequency distribution of Fe average charge states, 0.25 binning.

- For samples at the higher speeds, which are expected to be predominately $\mathrm{CH}$-associated solar wind, the average $\mathrm{Fe}<\mathrm{Q}>$ is only slightly lower (by a fraction of a charge state) than that of the slow solar wind.

- For samples at the higher speeds, which are expected to be predominately $\mathrm{CH}$-associated solar wind, the spread in the distribution of $\mathrm{Fe}<\mathrm{Q}>$ values narrows, with the standard deviation of the $\mathrm{Fe}<\mathrm{Q}>$ value in the higher speed bins becoming half of that observed in the lower speed bins. (See upper insert in Fig. 5.) 
Table 2. Coronal hole-associated and slow solar wind: iron average charge states binned by proton speed.

\begin{tabular}{ccccccc}
\hline$V p$ Bin & Sample size & $\left\langle V p>_{\text {bin }}\right.$ & Std Dev $<V p>_{\text {bin }}$ & Fe $<\mathrm{Q}>_{\text {bin }}$ & Std Dev Fe $<Q>_{\text {bin }}$ & Fe $<Q>$ Range Min-Max \\
\hline $250-300$ & 863 & 288.4 & 8.4 & 9.53 & .43 & $8.5-11.8$ \\
$300-350$ & 4705 & 322.6 & 14.6 & 9.51 & .43 & $8.1-11.4$ \\
$350-400$ & 3501 & 373.2 & 14.7 & 9.53 & .48 & $8.0-11.4$ \\
$400-450$ & 2919 & 422.0 & 14.3 & 9.43 & .45 & $8.4-10.9$ \\
$450-500$ & 2068 & 473.7 & 14.5 & 9.30 & .42 & $8.4-10.9$ \\
$500-550$ & 1545 & 522.6 & 14.4 & 9.18 & .37 & $8.4-10.6$ \\
$550-600$ & 1274 & 574.4 & 14.8 & 9.17 & .32 & $8.3-10.2$ \\
$600-650$ & 1407 & 624.8 & 14.1 & 9.20 & .28 & $8.3-10.3$ \\
$650-700$ & 814 & 668.0 & 12.7 & 9.25 & .24 & $8.6-10.0$ \\
$700-750$ & 144 & 718.1 & 13.1 & 9.17 & .24 & $8.7-9.9$ \\
$750-800$ & 15 & 771.9 & 16.4 & 8.7 & .10 & $8.6-8.9$ \\
\hline $250-800$ & 19255 & 434.3 & 112.5 & 9.39 & .44 & $8.0-11.8$ \\
\hline
\end{tabular}

\subsection{Stream interfaces and solar origins: case study of Carrington Rotation 2064}

We next present a case study of Carrington Rotation Number 2064. During this period, which occurs from 3 December to 30 December 2007, for STEREO A, there are no reported ICMEs or shocks (Jian, 2009) at STEREO A, although ICMEs were observed at Wind and STEREO B (Kilpua et al., 2009c). For CR 2064, $\left\langle V p>_{\mathrm{CR} 2064}=457 \pm 123 \mathrm{~km} / \mathrm{s}\right.$ for STEREO A, which is typical for this data set, and $\mathrm{Fe}$ $<\mathrm{Q}>_{\mathrm{CR} 2064}$ was $9.45 \pm 0.37$, which was in the mid-range of the values observed in the survey. Solar wind high-speed streams at STEREO A and STEREO B had similar proton speed and $\mathrm{Fe}<\mathrm{Q}>$ profiles, as seen in the top two panels of Fig. 6, implying relatively quasi-stable source coronal hole conditions.

In the remainder of Fig. 6 we present an overview of Carrington Rotation 2064 from the in situ solar wind perspective at STEREO A. In the third panel, the time profile of the solar wind speed as measured by STA PLASTIC for protons, alphas, and the minor ions oxygen and iron is provided. (The speeds for Helium were restricted to measurements in the small geometrical factor portion of PLASTIC, resulting in some gaps here.) As expected, the speeds for all species are very similar within the slow solar wind, while the alphas and minor ions show higher speeds relative to the protons in the high-speed solar wind. This is further illustrated in the fifth panel, which provides the STA speed differential derived between iron and protons $\left(V_{\mathrm{Fe}}-V p\right)$. As seen here, the speed differential parameter is another feature that appears to be useful in distinguishing slow from coronal hole-associated solar wind. This has been previously noted by WimmerSchweingruber et al. (1999), who used differential streaming of alpha particles relative to protons as one of five potential criteria for defining the stream interface. In their study, the helium speed difference was seen in 6 of the 11 CIR/SIRs selected (that is, 55\%).
The fourth panel presents the STA proton bulk flow directions (east-west and north-south) in the RTN frame of reference. The aberration effect from the spacecraft motion has been removed from both sets of flow angles. As explained in the review by Gosling (1996), east-west deflections are an expected pattern at stream interaction regions. First the slow solar wind deflects westward as it is overtaken by the forward wave bounding the stream interaction region, and then the fast solar wind deflects eastward as it encounters the reverse wave. (East and west directions are given by Gosling, 1996, in the sense of planetary motion.) That pattern is seen here as the observed east-west flow angles first deflect westward up to $+5.6^{\circ}$ then eastward to $-9.8^{\circ}$ for SI $1 ;+7.5^{\circ}$ to $-10.2^{\circ}$ for SI-2a; and then $+4.3^{\circ}$ to $-2.7^{\circ}$ for SI- $2 \mathrm{~b}$ (hourly averages for the flow angles).

In the bottom two panels are two common parameters used for determining stream interfaces: total pressure and entropy. The maximum of the total pressure (magnetic field, ions, and electrons) indicates where the forces at the two sides of the interface are equal and in opposite direction (Jian, 2009). Changes in entropy, which combines information from the proton kinetic temperature and density, have also been used for identifying stream interfaces by Simunac (2009), Burton et al. (1999), and Burlaga et al. (1990). At the bottom of the plots, the slow-to-fast stream interfaces for STA are shown (SI-1, SI-2a, SI-2b) as determined using the entropy method described by Simunac (2009).

Ballistic back mapping using the PLASTIC proton speed and the STEREO A spacecraft location during CR 2064 is used to provide an estimate of the originating solar longitude of the solar wind parcels. (This technique assumes the solar wind speed has remained constant from the Sun to the spacecraft, and is only an approximation.)

The STEREO A back-mapped data are shown in Fig. 7, and include the back-mapped solar wind parameters $\mathrm{Vp}$ and $\mathrm{Fe}<\mathrm{Q}>$, as well as the GONG synoptic coronal hole map, and the STEREO SECCHI EUVI synoptic map for this CR. 


\section{STEREO Solar Wind Parameters CR 2064}
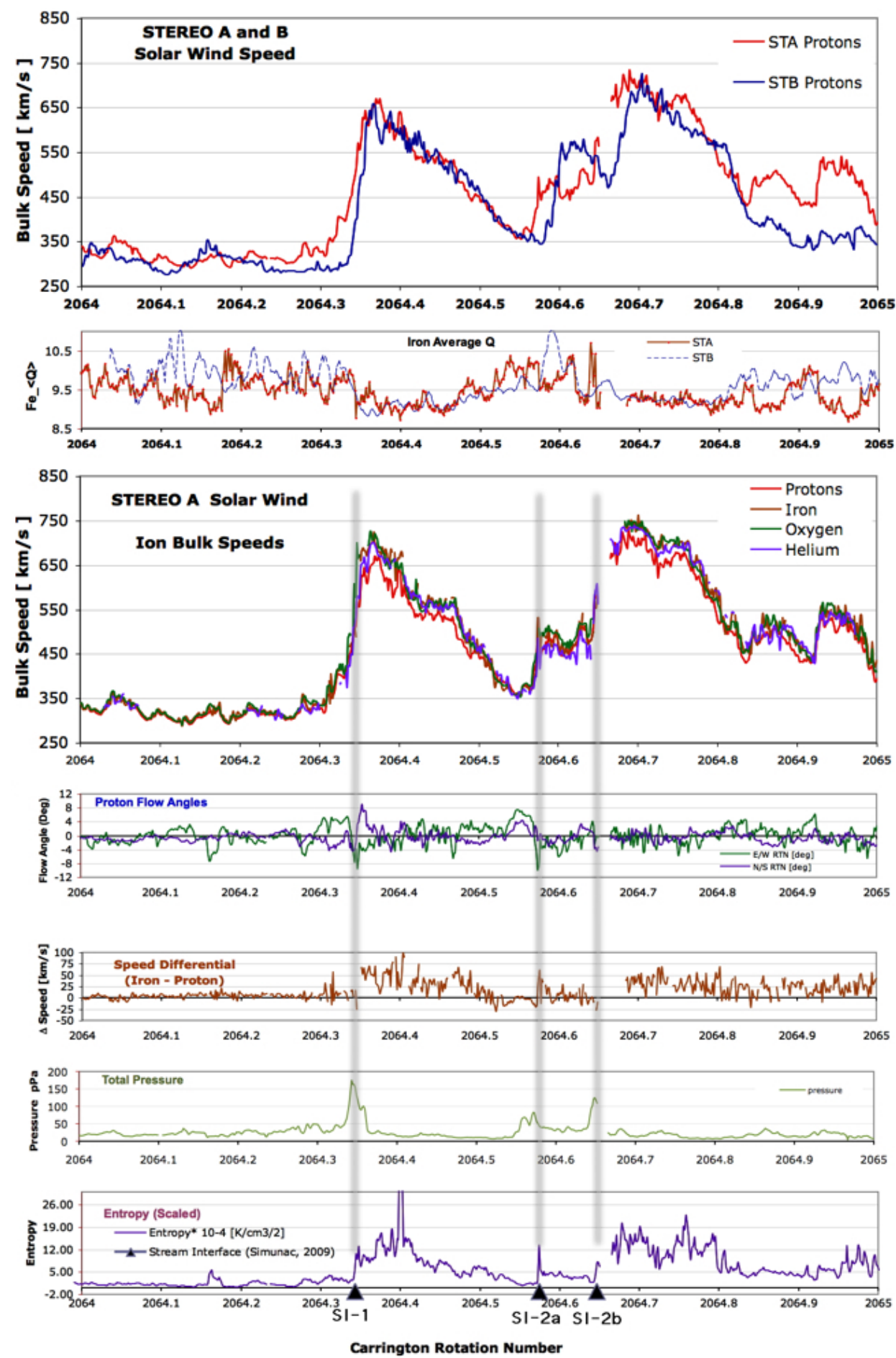

Fig. 6. Solar wind overview of Carrington Rotation Number 2064 as observed by STEREO A (STA) and STEREO B (STB). From top to bottom: bulk speed profile for protons during Carrington Rotation Number 2064 observed at STEREO A (3-30 December 2007) and STEREO B (30 November-27 December 2007); average Fe charge state for STEREO A and B; Ion bulk speeds observed by STEREO A for protons, helium, oxygen, and iron; Proton bulk flow directions (E/W, N/S) in the RTN frame of reference at STEREO A; Speed differential between iron and protons at STEREO A; Total pressure (magnetic and plasma - ion and electron) at STEREO A; Entropy (scaled) and Stream interfaces (SI-1, SI-2a, SI-2b) as determined by Simunac (2009) at STEREO A. 
The EUVI map shows the coronal holes as darker regions. The GONG map indicates the coronal holes, as well as their magnetic polarity. GONG also provides the location of the neutral line. Superimposed on the GONG plot is the projection of the trajectory of the STEREO A spacecraft. Below the GONG plot is a panel with the IMPACT in situ magnetic field radial component signage, mapped back using the proton speed. The GONG synoptic map indicates consistency between the location of the STEREO trajectory in relation to the neutral line, and IMPACT magnetic field polarity.

The slow-to-fast stream interfaces determined from the entropy method in Fig. 6 (SI-1, SI-2a, SI-2b), are shown here in the back-mapped configuration. (Note that time goes from right to left in the Carrington Longitude format.) The arrows superimposed on the SECCHI image indicate the backmapped longitudes for the SIs. The point of the arrow is at the presumed $\mathrm{CH}$ feature providing the fast wind, based on polarity (this back-mapping technique does not actually provide the latitude of the solar source).

The first stream interface, SI-1, maps back to the leading edge of an equatorial coronal hole (seen clearly here in the SECCHI synoptic map). S2-a and S2-b map back to extensions of the south polar hole. The equatorial and polar holes are of opposite polarity, consistent with the IMPACT magnetic field sense.

When including the in situ magnetic field information and the back-mapped solar features, some of the variations seen in the $\mathrm{Fe}<\mathrm{Q}>$ within the slow solar wind may be linked to multiple stream interface crossings (see CIR 7 of WimmerSchweingruber et al., 1999). Other variations might be linked to non-uniform compositional signatures at HCS crossings (see Suess et al., 2009). These are items for future study.

\section{Discussion and summary}

In this paper we have presented temporal trends in the solar wind proton speeds and iron average charge states in the solar wind for the past two years. Comparisons of $\mathrm{Fe}<\mathrm{Q}>$ with $V p$ bins and solar wind flow type have been presented. We have also provided a case study (CR 2064) with a comparison of multiple parameters in defining stream interfaces, and their map-backed origins on the Sun.

The majority of the ICME periods identified during this period (Jian, 2009; Kilpua et al., 2009a, b, c) exhibit iron average charge states that fall within the range observed for slow solar wind. The exception was the event periods of $22-$ 24 May 2007. These results have been discussed in terms of the solar minimum conditions.

The values of the iron average charge state determined for slow and coronal hole-associated solar wind are consistent with values derived from the long-accumulation time studies of earlier authors (e.g., von Steiger et al., 2000). However, with the one-hour sampling available here, it is evident that there are modest but measurable variations in the measured

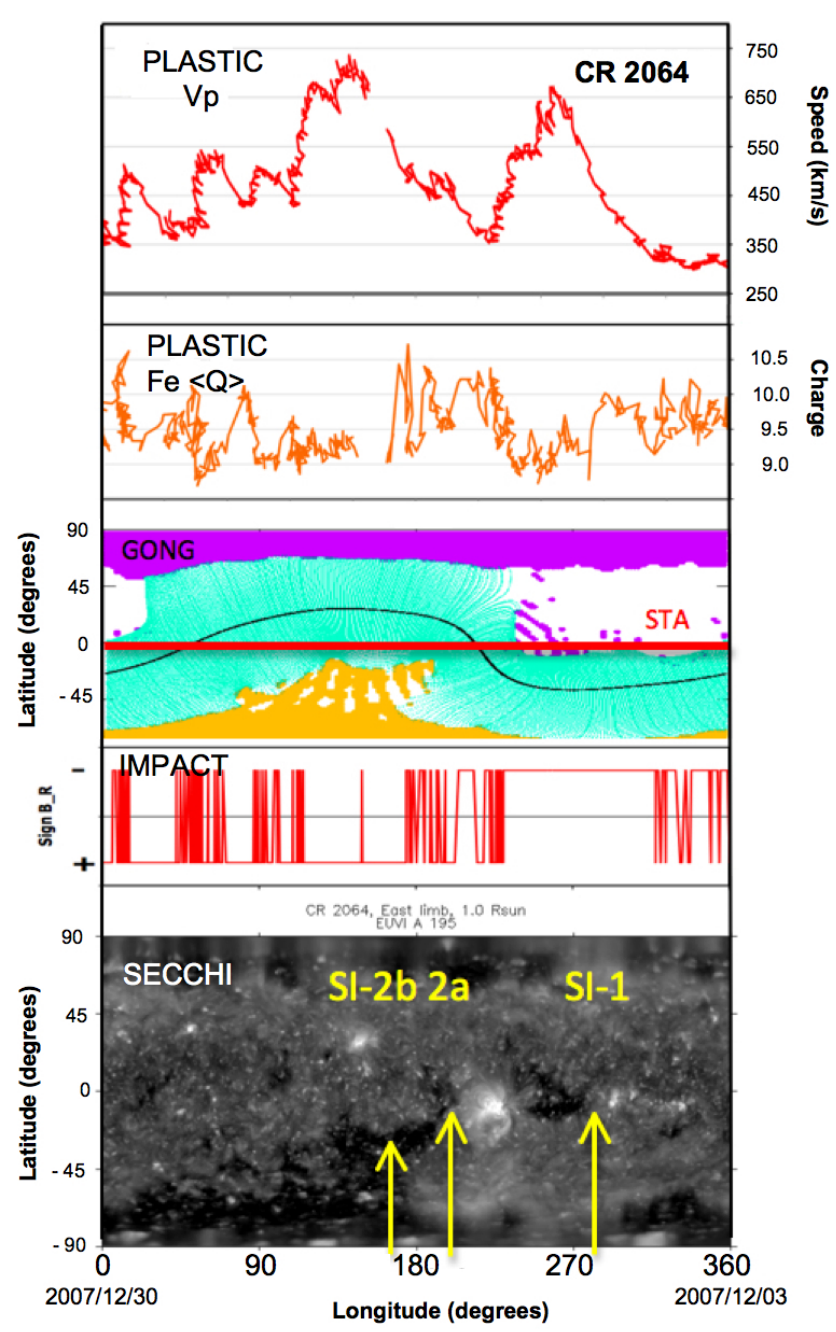

Fig. 7. Mapping solar wind to solar origins. From top to bottom: STEREO A (STA) PLASTIC proton speeds back-mapped to their originating Carrington Rotation (CR) number, or equivalently, Carrington Longitude; PLASTIC Fe $<\mathrm{Q}>$; GONG coronal hole synoptic map for CR 2064, showing the coronal holes, their magnetic polarity, and the current sheet as a function of both latitude and longitude, and the STEREO A projected trajectory superimposed; Signage of the radial component of the STEREO A IMPACT/MAG interplanetary magnetic field, indicative of the in-situ magnetic polarity; Solar synoptic map from SECCHI EUVI. Vertical arrows indicate the location of stream interfaces SI-1, SI-2a, and SI-2b identified by Simunac (2009). Note that time is reversed in this format.

average charge states observed between and within flows, for both slow and coronal hole-associated solar wind. These population variations are statistically smaller for the highspeed solar wind (coronal hole-associated). We again note here that we have not yet separated slow solar wind into subcategories.

Returning to the apparent temporal variation in the $\mathrm{Fe}$ $<$ Q $>$ observed in Fig. 1 and Table 1: the overall decrease in iron charge states over the survey time interval may be due to the one or more of the following factors: 
1. The data have not been normalized by flow type, which biases the average toward the predominance (by time coverage) of a given flow type observed in the ecliptic within a given averaging interval. In the case of slow solar wind, the progressive decrease in the number of active regions during the course of the survey period decreases the contributions by slow solar wind associated with solar activity (Schwenn, 2006). The first part of the survey likely includes both types of slow solar wind, but as the solar minimum progresses, the slow solar wind is more likely originating solely (or nearly so) from the relatively quiescent helmet streamer region.

2. The charge state distribution in the $\mathrm{CH}$-solar wind may have changed due to a temporal change in the coronal holes in the approach to solar minimum.

3. The type of coronal-hole sources from which the solar wind emanated has changed over the survey period. Isolated coronal holes and polar extensions were primary sources for the recurrent high speeds observed in the in-ecliptic solar wind at the start of the survey period; while the polar holes themselves were the high-speed sources toward the end of the survey (Fig. 2).

With regard to points 2) and 3), it is important to note that differences in charge state distributions, and in elemental abundances, have been observed in solar wind originating from different coronal holes in a limited number of studies. Galvin and Gloeckler (1997) studied eight solar rotations of three long-lived coronal holes using in-ecliptic measurements by Wind/STICS. These observations were taken in January-July 1995, about a year before the last solar minimum. Two of the recurrent high-speed streams were traced to coronal holes of negative magnetic polarity, one of these a near equatorial, isolated coronal hole, the other an extension (sometimes quasi-detached) of the south polar hole. The third recurrent high-speed stream originated from a near-equatorial coronal hole of positive polarity. Data over the 8 rotations were separated by stream, but then summed together for statistics. The two streams linked to holes of negative polarity exhibited a dominant iron charge state of +9 , while the one stream linked to the isolated hole of positive polarity exhibited dominant $\mathrm{Fe}$ charge states +9 and +10 . Modest differences in the charge state distributions in the north polar hole versus the south polar hole were also reported by Ulysses, although in that case temporal affects could not be ruled out.

Differences in the heliographic latitude being sampled within a given coronal hole have been shown to exhibit systematic variations in the charge state distributions, at least for a polar hole and its extensions. Galvin and Gloeckler (1997) determined an unambiguous latitudinal variation in the $\mathrm{CH}$ charge state distributions of iron and silicon in the north polar pass of Ulysses during last solar minimum (observations from March 1995 through December 1996) and spacecraft latitudes of $20^{\circ} \mathrm{N}$ to $80^{\circ} \mathrm{N}$. Both iron and silicon exhibited a latitudinal gradient in their respective charge state ratios. The measurements were taken for both the ascent and descent trajectories of the space probe, thereby establishing the observed trends were spatial, not temporal, within the coronal hole.

To address the above possibilities, follow up studies will include categorizing the dataset by flow type, allowing temporal trends to be established separately for the "slow" versus coronal hole-associated solar winds. The $\mathrm{CH}$ wind will be further categorized by coronal hole characteristics, and the slow solar wind by degree of solar activity. We have also not distinguished HCS in the slow solar wind data set. This is a separate study.

In summary, the first two years of the STEREO mission has coincided with the quietest period in the Sun since in situ solar wind measurements became available. This has provided an exceptional opportunity for determining quiet solar wind minor ion trends in fast and slow solar wind, boundaries such as stream interfaces, and transients during minimum conditions.

Acknowledgements. The authors thank their colleagues in the STEREO mission, including APL and NASA. We especially thank Russ Howard and the SECCHI team for the use of the EUVI synoptic map. The PLASTIC portions of this work were funded under NASA STEREO NAS5-00132 at UNH.

This work utilizes data obtained by the Global Oscillation Network Group (GONG) program, managed by the National Solar Observatory, which is operated by AURA, Inc., under a cooperative agreement with the National Science Foundation. The data were acquired by instruments operated by the Big Bear Solar Observatory, High Altitude Observatory, Learmonth Solar Observatory, Udaipur Solar Observatory, Instituto de Astrofisica de Canarias, and Cerro Tololo Interamerican Observatory.

Topical Editor R. Forsyth thanks R. Bucik and another anonymous referee for their help in evaluating this paper.

\section{References}

Aellig, M. R., Grunwaldt, H., Bochsler, P., Wurz, P., Hefti, S., Kallenbach, R., Ipavich, F. M., Axford, W. I., Balsiger, H., Burgi, A., Coplan, M. A., Galvin, A. B., Geiss, J., Gliem, F., Gloeckler, G., Hilchenbach, M., Hovestadt, D., Hsieh, K. C., Klecker, B., Lee, M. A., Livi, S., Managadze, G. G., Marsch, E., Mobius, E., Neugebauer, M., Reiche, K.-U., Scholer, M., Verigin, M. I., and Wilken, B.: Iron freeze-in temperatures measured by SOHO/CELIAS/CTOF, J. Geophys. Res., 103, 17215-17222, 1998.

Burlaga, L. F., Mish, W. H., and Whang, Y. C.: Coalescence of recurrent streams of different sizes and amplitudes, J. Geophys. Res., 95, 4247-4255, 1990.

Burton, M. E., Neugebauer, M., Crooker, N. U., von Steiger, R., and Smith, E. J.: Identification of trailing edge solar wind stream interfaces: A comparison of Ulysses plasma and composition measurements, J. Geophys. Res., 104, 9925-9932, 1999. 
Cranmer, S. R.: Coronal Holes and the high-speed solar wind, Space Sci. Rev., 101, 229-291, doi:10.1023/A:1020840004535, 2002.

Daoudi, H., Blush, L. M., Bochsler, P., Galvin, A. B., Giammanco, C., Karrer, R., Opitz, A., Wurz, P., Farrugia, C., Kistler, L. A., Popecki, M. A., Möbius, E., Singer, K., Klecker, B., Wimmer-Schweingruber, R. F., and Thompson, B.: The STEREO/PLASTIC response to solar wind ions (Flight measurements and models), Astrophys. Space Sci. Trans., 5, 1-13, 2009, http://www.astrophys-space-sci-trans.net/5/1/2009/.

Fisk, L. A., Schwadron, N. A., and Zurbuchen, T. H.: On the slow solar wind, Space Sci. Rev., 86, 51-60, doi:10.1023/A:1005015527146, 1998.

Galvin, A. B., Ipavich, F. M., Gloeckler, G., von Steiger, R., and Wilken, B.: Silicon and oxygen charge state distributions and relative abundances in the solar wind measured by SWICS on Ulysses, in: Solar Wind Seven, COSPAR Colloq. Ser., vol. 3, edited by: Marsch, E. and Schwenn, R., pp. 337-340, Pergamon, Tallytown, N.Y., 1992.

Galvin, A. B.: Minor Ion Composition in CME-Related Solar Wind, in: Coronal Mass Ejections, Geophysical Monograph, 99, edited by: Crooker, N., Joselyn, J. A., and Feynman, J., American Geophysical Union, 253-260, 1997.

Galvin, A. B. and Gloeckler, G.: Charge State Composition in Coronal Hole and CME Related Solar Wind: Latitudinal Variations Observed by Ulysses and WIND, 31st ESLAB Symposium, Correlated Phenomena at the Sun, in the Heliosphere and in Geospace, ESA SP-415, pp. 323-326, 1997.

Galvin, A. B., Kistler, L. M., Popecki, M. A., Farrugia, C. J., Simunac, K. D. C., Ellis, L., Mobius, E., Lee, M. A., Boehm, M., Carroll, J., Crashaw, A., Conti, M., Demaine, P., Ellis, S., Gaidos, J. A., Googins, J., Granoff, M., Gustafson, A., Heirtzler, D., King, B., Knauss, U., Levasseur, J., Longworth, S., Singer, K., Turco, S., Vachon, P., Vosbury, M., Widholm, M., Blush, L. M., Karrer R., Bochsler, P., Daoudi, H., Etter, A., Fischer, J., Jost, J., Opitz, A., Sigrist, M., Wurz, P., Klecker, B., Ertl, M., Seidenschwang, E., Wimmer-Schweingruber, R. F., Koeten, M., Thompson, B., and Steinfeld, D.: The Plasma and Suprathermal Ion Composition (PLASTIC) Investigation on the STEREO Observatories, Space Sci. Rev., 136, 437-486, doi:10.1007/s11214007-9296-x, 2008.

Geiss, J., Gloeckler, G., and von Steiger, R.: Origin of the solar wind from composition data, Space Sci. Rev., 72, 49-60, 1995.

GONG: http://gong.nso.edu/data/magmap/, 2009.

Gosling, J. T.: Corotating and Transient Solar Wind Flows in Three Directions, Annu. Rev. Astron. Astrophys., 34, 35-73, 1996.

Ipavich, F. M., Galvin, A. B., Geiss, J., Ogilvie, K. W., and Gliem, F.: Solar Wind Iron and Oxygen Charge States and Relative Abundances Measured by SWICS on Ulysses, in: Solar Wind Seven, COSPAR Colloq. Ser., vol. 3, edited by: Marsch, E. and Schwenn, R., 369-374, Pergamon, Tallytown, N.Y., 1992.

Isenberg, P. A.: Heating of coronal holes and generation of the solar wind by ion-cyclotron resonance, Space Sci. Rev., 95, 119-131, doi:10.1023/A:1005287225222, 2001.

Jian, L.: Level 3 Results of STEREO IMPACT/PLASTIC, http:// www-ssc.igpp.ucla.edu/forms/stereo/stereo_level_3.html, 2009.

Jian, L. K., Russell, C. T., Luhmann, J. G., Galvin, A. B., and MacNeice, P. J.: Multi-Spacecraft Observations: Stream Interactions and Associated Structures, Solar Phys., doi:10.1007/s11207-
009-9445-3, in press, 2009.

Kilpua, E. K. J., Luhmann, J. G., Gosling, J., Li, Y., Elliott, H., Russell, C. T., Jian, L., Galvin, A. B., Larson, D., Schroeder, P., Simunac, K., and Petrie, G.: Small solar wind transients and their connection to the large-scale coronal structure, Solar Phys., 256, 327-344, doi:10.1007/s11207-009-9366-1, 2009a.

Kilpua, E. K. J., Pomoell, J., Vainio, R., Luhmann, J., Li, Y., Vourlidas, A., Schroeder, P., Galvin, A. B., and Simunac, K.: STEREO observations of interplanetary coronal mass ejections and prominence de?ection during solar minimum period, Ann. Geophys., in review, 2009b.

Kilpua, E. K. J., Liewer, P., Farrugia, C., Luhmann, J., Möstl, C., Li, Y., Liu, Y., Lynch, B., Vourlidas, A., Acuna, M., Galvin, A. B., Larson, D., Russell, C., and Sauvaud, J.: Multispacecraft observations of magnetic clouds and their solar origins May 1923, 2007, Solar Phys., 254, 325-344, doi:10.1007/s11207-0089300-y, 2009c.

Leitner, M., Farrugia, C. J., Galvin, A., Biernat, H. K., and Osherovich, V. A.: The solar wind Quasi-Invariant observed by STEREO A and B at solar minimum 2007, and comparison with two other minima, Solar Phys., doi:10.1007/s11207-009-9412-z, in press, 2009.

Lemen, J. R., Acton, L. W., Alexander, D., Galvin, A. B., Harvey, K. L., Hoeksema, J. T., Zhao, X., and Hudson, H. S.: Solar Identification of Solar-Wind Disturbances observed at Ulysses, in: Solar Wind Eight, edited by: Winterhalter, D., Gosling, J. T., Habbal, S. R., Kurth, W. S., and Neugebauer, M.: Proc. of the Eighth International Solar Wind Conference, Dana Point, CA, June 1995, AIP Conference Proceedings, Woodbury, NY, pp. 92 95, 1996.

Lindblad, B. A.: Coronal sources of high-speed plasma streams in the solar wind during the declining phase of solar cycle 20, Astrophys. Space Sci., 170, 55-61, doi:10.1007/BF00652645, 1990.

Luhmann, J. G., Curtis, D. W., Schroeder, P., McCauley, J., Lin, R. P., Larson, D. E., Bale, S. D., Sauvaud, J.-A., Aoustin, C., Mewaldt, R. A., Cummings, A. C., Stone, E. C., Davis, A. J., Cook, W. R., Kecman, B., Wiedenbeck, M. E., von Rosenvinge, T., Acuna, M. H., Reichenthal, L. S., Shuman, S., Wortman, K. A., Reames, D. V., Mueller-Mellin, R., Kunow, H., Mason, G. M., Walpole, P., Korth, A., Sanderson, T. R., Russell, C. T., and Gosling, J. T.: STEREO IMPACT Investigation Goals, Measurements, and Data Products Overview, Space Sci. Rev., 136, 117 184, doi:10.1007/s11214-007-9170-x, 2008.

Luhmann, J. G., Lee, C. O., Li, Yan, Arge, C. N., Galvin, A. B., Simunac, K., Russell, C. T., Howard, R .A., and Petrie, G.: Solar wind sources in the late declining phase of cycle 23: Effects of the weak solar polar filed on high speed streams, Solar Phys., 256, 285-305, doi:10.1007/s11207-009-9354-5, 2009.

McComas, D. J., Ebert, R. W., Elliott, H. A., Goldstein, B. E., Gosling, J. T., Schwadron, N. A., and Skoug, R. M.: Weaker solar wind from polar coronal holes and the whole Sun, Geophys. Res. Lett., 35, L18103, doi:10.1029/2008GL034896, 2008.

Möstl, C., Farrugia, C. J., Biernat, H. K., Leitner, M., Kilpua, E. K. J., Galvin, A. B., and Luhmann, J. G.: Optimized Grad-Shafranov reconstruction of a small magnetic cloud using STEREO-WIND observations, Solar Phys., 256, 427-441, doi:10.1007/s11207-009-9360-7, 2009.

Reinard, A.: Comparison of interplanetary CME charge state com- 
position with CME-associated flare magnitude, The Astrophys. J., 620, 501-505, 2005.

Reinard, A.: Analysis of interplanetary coronal mass ejection parameters as a function of energetics, source location, and magnetic structure, The Astrophys. J., 682, 1289-1305, 2008.

Richardson, I. G. and Cane, H. V.: Identification of interplanetary coronal mass ejections at $1 \mathrm{AU}$ using multiple solar wind plasma composition anomalies, J. Geophys. Res., 109, A09104, doi:10.1029/2004JA010598, 2004.

Schwenn, R.: Solar wind sources and their variations over the solar cycle, Space Sci. Rev., 124, 51-76, doi:10.1007/s11214-0069099-5, 2006.

Sheeley Jr., N. R., Wang, Y.-M., Hawley, S. H., Brueckner, G. E., Dere, K. P., Howard, R. A., Koomen, M. J., Korendyke, C. M., Michels, D. J., Paswaters, S. E., Socker, D. G., St. Cyr, O. C., Wang, D., Lamy, P. L., Llebaria, A., Schwenn, R., Simnett, G. M., Plunkett, S., and Biesecker, D. A.: Measurements of Flow Speeds in the Corona between 2 and $30 \mathrm{R}$ sub sun, Astrophys. J., 484, 472-478, 1997.

Simunac, K. D. C.: Solar Wind Stream Interfaces: The Importance of Time, Longitude, and Latitude Separation between Points of Observation, Ph.D. dissertation, University of New Hampshire, Durham, NH, 2009.

Simunac, K. D. C., Kistler, L. M., Galvin, A. B., Lee, M., Popecki, M. A., Farrugia, C., Möbius, E., Blush, L. M., Bochsler, P., Wurz, P., Klecker, B., Wimmer-Schweingruber, R. F., Thompson, B., Luhmann, J. G., Russell, C. T., and Howard, R. A.: In situ observations of solar wind stream interface evolution, Solar Phys., doi:10.1007/s11207-009-9393-y, in press, 2009.
Suess, S. T., Ko, Y.-K., von Steiger, R., and Moore, R. L.: Quiescent current sheets in the solar wind and origins of slow wind, J. Geophys. Res., 114, A04103, doi:10.1029/2008JA013704, 2009.

Von Steiger, R., Schwadron, N. A., Fisk, L. A., Geiss, J., Gloeckler, G., Hefti, S., Wilken, B., Wimmer-Schweingruber, R. R., and Zurbuchen, T. H.: Composition of quasi-stationary solar wind flows from Ulysses/Solar Wind Ion Composition Spectrometer, J. Geophys. Res., 105(A12), 27217-27238, 2000.

Wimmer-Schweingruber, R. F.: The composition of the solar wind, Adv. Space Res., 30, 23-32, 2002.

Wimmer-Schweingruber, R. F., von Steiger, R., and Parli, R.: Solar wind stream interfaces in corotating interaction regions: SWICS/Ulysses results, J. Geophys. Res., 102, 17407-17417, 1997.

Wimmer-Schweingruber, R. F., von Steiger, R., and Paerli, R.: Solar wind stream interfaces in corotating interaction regions: New SWICS/Ulysses results, J. Geophys. Res., 104, 9933-9945, 1999.

Zurbuchen, T. H., Hefti, S., Fisk, L. A., Gloeckler, G., and von Steiger, R.: The transition between fast and slow solar wind from composition data, Space Sci. Rev., 87, 353-356, 1999. 